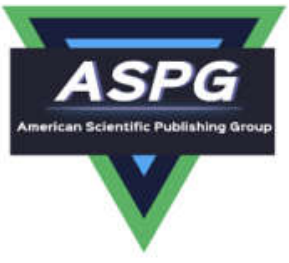

\title{
A Review On Some Concepts and Open Questions In Neutrosophic Algebraic Structures
}

\author{
Rozina Ali \\ Independent researcher, Cairo, Egypt. \\ e-mail:rozyyy123n@gmail.com
}

\begin{abstract}
The aim of this paper is to present a study about recent progressions in the study of neutrosophic algebraic structures. Also, it lists the most interesting open questions about neutrosophic algebraic structures such as neutrosophic rings, refined neutrosophic rings and $n$-refined neutrosophic rings.

On the other hand, a study on neutrosophic ring of complex numbers has been presented, with many Smarandache-Kandasamy open problems about the properties of these numbers, especially algebraic ones.
\end{abstract}

Keywords: Neutrosophic ring, refined neutrosophic ring, $n$-refined neutrosophic ring, neutrosophic complex number, AH-ideals.

\section{Introduction}

Neutrosophy is a new kind of logic proposed by Smarandache to generalize the intuitionistic fuzzy logic [1], where he defined the concept of neutrosophic sets. These sets played a basic role in different field of applied mathematics such as pattern recognition [2], machine learning [3], health care [4], computer science [5], and statistics [6]. Many interesting applications of neutrosophy can be found in $[29,30,35,37,40-43,45,47,50]$.

In the last few years, neutrosophic sets became a useful tool in the study of pure mathematics like topology [7], algebraic equations [8], number theory [9], and it was used in the study of algebraic structures such as modules [10,11], spaces $[12,13]$, groups $[14,15]$, and matrix theory $[51,55,56]$.

The concept of neutrosophic ring was defined firstly by Kandasamy and Smarandache in [16], and then it was studied by Agboola et.al in [17]. Recently, many generalizations were defined and handled such as refined neutrosophic rings [18,19], and n-refined neutrosophic rings [20]. 
These rings were studied widely, where Kandasamy et.al. have determined the form of triplets group [21], idempotents [22], and semi idempotents [23]. Recently, many researchers concentrate their efforts to study these structures, where AH-substructures were defined in [24,25], refined idempotents [26], and AH-homomorphisms [27].

An important generalization was presented in [28] by Smarandache and Kandasamy, where they defined neutrosophic complex numbers ring, and neutrosophic finite complex numbers.

Through this paper, we study the basic definitions and theorems about neutrosophic rings, refined neutrosophic rings, and $n$-refined neutrosophic rings. In addition, we study some of important open questions about those rings. These questions are still open, their answers will have a huge effect in theoretical progression in many related fields. Also, we add some examples to ensure and clarify these concepts in general.

\section{Neutrosophic rings}

\section{Definition 2.1: [24]}

Let $R$ be a ring, $I$ be the indeterminacy with property $I^{2}=I$, then the neutrosophic ring is defined as follows:

$R(I)=\{a+b I ; a, b \in R\}$.

\section{Definition 2.2: [24]}

Let $R(I)$ be a neutrosophic ring, it is called commutative if and only if $x y=y x \forall x, y \in R(I)$.

Definition 2.3: [17]

(a) Let $x$ be any element in $\mathrm{R}(\mathrm{I})$, it is called idempotent if and only if $x^{2}=x$.

(b) $x, y$ are called a duplet if and only if $x y=y x=x$.

Definition 2.4: [17]

Let $R(I)$ be a neutrosophic ring, a non-empty subset $\mathrm{P}$ of $\mathrm{R}(\mathrm{I})$ is called a neutrosophic ideal if

(a) $\mathrm{P}$ is a neutrosophic subring of $\mathrm{R}(\mathrm{I})$

(b) $r x \in P$ for every $x \in P$ and $r \in R(I)$.

\section{Definition 2.5:[24]}

Let $R(I)$ be a neutrosophic ring and $P=P_{0}+P_{1} I=\left\{a_{0}+a_{1} I ; a_{0} \in P_{0}, a_{1} \in P_{1}\right\}$.

(a)We say that $P$ is an AH-ideal if $P_{0}, P_{1}$ are ideals in the ring R.

(b)We say that $P$ is an AHS-ideal if $P_{0}=P_{1}$.

(c) The AH-ideal $P$ is called null if $P_{0}, P_{1} \in\{R, O\}$. 


\section{Theorem 2.1:[24]}

Let $R(I)$ be a neutrosophic ring and $P=P_{0}+P_{1} I$ be an AH-ideal, then $P$ is not a neutrosophic ideal in general by the classical meaning.

\section{Definition 2.6:[24]}

Let $R(I)$ be a neutrosophic ring and $P=P_{0}+P_{1} I$ be an AH-ideal, we define the AH-factor as: $R(I) / P=$ $R / P_{0}+R / P_{1} I$.

\section{Definition 2.7:[24]}

Let $R(I)$ be a neutrosophic ring and $P=P_{0}+P_{1} I$ be an AH-ideal then $R(I) / P$ is a ring with the following two binary operations:

$$
\begin{aligned}
& {\left[\left(x_{0}+P_{0}\right)+\left(y_{0}+P_{1}\right) I\right]+\left[\left(x_{1}+P_{0}\right)+\left(y_{1}+P_{1}\right) I\right]=} \\
& {\left[\left(x_{0}+x_{1}+P_{0}\right)+\left(y_{0}+y_{1}+P_{1}\right) I\right] .} \\
& {\left[\left(x_{0}+P_{0}\right)+\left(y_{0}+P_{1}\right) I\right] \times\left[\left(x_{1}+P_{0}\right)+\left(y_{1}+P_{1}\right) I\right]=\left[\left(x_{0} \times x_{1}+P_{0}\right)+\left(y_{0} \times y_{1}+P_{1}\right) I\right] .}
\end{aligned}
$$

\section{Definition 2.8:[24]}

Let $R(I), T(J)$ be two neutrosophic rings and the map $f: R(I) \rightarrow T(J)$ we say that $f$ is a neutrosophic AHShomomorphism if:

The restriction of the map $f$ on $R$ is a ring homomorphism from $R$ to $T$ i.e. $f_{R}: R \rightarrow T$ is homomorphism and

$f(a+b \mathrm{I})=f_{R}(a)+f_{R}(b) J$.

We say that $R(I), T(J)$ are AHS-isomorphic neutrosophic rings if there is a neutrosophic AHShomomorphism $f: R(I) \rightarrow T(J)$ which is a bijective map i.e. ( $\mathrm{R} \cong T$ ), we say that $\mathrm{f}$ is a neutrosophic AHS-isomorphism.

\section{Theorem 2.2:[24]}

Let $R(I), T(J)$ be two neutrosophic rings and $f: R(I) \rightarrow T(J)$ is a neutrosophic ring AHS-homomorphism, let $P=P_{0}+P_{1} I$ be an AH-ideal of R(I) and $Q=Q_{0}+Q_{1} J$ be an AH-ideal of $T(J)$, we have:

(a) $f(P)$ is an AH-ideal of $f(R(I))$. 
(b) $f^{-1}(Q)$ is an AH-ideal of $R(I)$.

(c) If $P$ is an AHS-ideal of $R(I)$, then $f(P)$ is an AHS-ideal of $f(R(I))$.

(d) $A H-\operatorname{kerf}=\operatorname{kerf} f_{R}+\operatorname{ker} f_{R} I$ is an AHS-ideal; $f_{R}$ is the restriction of $f$ on the ring $R$.

(e) The AH-factor $R(I) /$ kerf is AHS - isomorphic to $f(R(I))$.

\section{Example 2.1:}

Let $\mathrm{R}(\mathrm{I})=Z_{6}(I), P_{0}=\{0,2,4\}, P_{1}=\{0,3\}$ be two ideals in $Z_{6}$, hence:

(a) $\mathrm{P}=P_{0}+P_{1} I=\{0,2,4,2+3 \mathrm{I}, 4+3 \mathrm{I}, 3 \mathrm{I}\}$ is an AH-ideal.

(b) $\mathrm{Q}=P_{1}+P_{1} I=\{0,3,3+3 I, 3 I\}$ is an AHS-ideal because $P_{1}=P_{1}$.

(c) We have: $R / P_{0}=\left\{P_{0}, 1+P_{0}\right\}$ and $R / P_{1}=\left\{P_{1}, 1+P_{1}, 2+P_{1}\right\}$; the corresponding AH-factor $R(I) / P=\left\{P_{0}+P_{1} I, P_{0}+\left(1+P_{1}\right) I, P_{0}+\left(2+P_{1}\right) I,\left(1+P_{0}\right)+P_{1} I,\left(1+P_{0}\right)+\left(1+P_{1}\right) I,\left(1+P_{0}\right)+\right.$ $\left.\left(2+P_{1}\right) I\right\}$

We should regard that $P_{0}=P_{0}+0 . I$ and $0=0+0 . I$.

(d)We can clarify the addition on the AH-factor $R(I) / P$ as:

$\left[P_{0}+\left(1+P_{1}\right) I\right]+\left[\left(1+P_{0}\right)+\left(2+P_{1}\right) I\right]=\left[(0+1)+P_{0}\right]+\left[(1+2)+P_{1}\right] \mathrm{I}=\left(1+P_{0}\right)+\left(3+P_{1}\right) \mathrm{I}=\left(1+P_{0}\right)+$ $P_{1} I$.

We can clarify the multiplication on the $\mathrm{AH}$-factor $R(I) / P$ as:

$\left[P_{0}+\left(1+P_{1}\right) I\right] \times\left[\left(1+P_{0}\right)+\left(2+P_{1}\right) I\right]=\left[(0 \times 1)+P_{0}\right]+\left[(1 \times 2)+P_{1}\right] I=P_{0}+\left(2+P_{1}\right) I$.

(e) We can see that $P \cap Q=\left(P_{0} \cap P_{1}\right)+\left(P_{1} \cap P_{1}\right) I=\{0\}+P_{1} I=\{0,3 I\}$ which it is an AH-ideal.

(f) $P+Q=\left(P_{0}+P_{1}\right)+\left(P_{1}+P_{1}\right) I=R+P_{1} I=\{0,1,2,3,4,5,3 I, 1+3 I, 2+3 I, \ldots \ldots 5+3 I\}$.

Open problem (1): Describe the algebraic structure of the group of units of a neutrosophic ring R(I)? What is its relation with the group of units of the ring $\mathrm{R}$ ?

\section{Refined neutrosophic rings}

\section{Definition 3.1:[44]}

The element I can be split into two indeterminacies $I_{1}, I_{2}$ with conditions: 
$\mathrm{I}_{1}^{2}=\mathrm{I}_{1}, I_{2}^{2}=I_{2}, I_{1} I_{2}=I_{2} I_{1}=I_{1}$.

\section{Definition 3.2:[44]}

If $\mathrm{X}$ is a set then $\mathrm{X}\left(I_{1}, I_{2}\right)=\left\{\left(a, b I_{1}, c I_{2}\right): a, b, c \in X\right\}$ is called the refined neutrosophic set generated by $X, I_{1}, I_{2}$.

\section{Definition 3.3:[44]}

Let $(R,+, \times)$ be a ring, $\left(R\left(I_{1}, I_{2}\right),+, \times\right)$ is called a 2-refined neutrosophic ring generated by $R, I_{1}, I_{2}$.

\section{Example 3.1:}

The refined neutrosophic ring of integers is $Z\left(I_{1}, I_{2}\right)=\left\{\left(a, b I_{1}, c I_{2}\right) ; a, b, c \in Z\right\}$.

\section{Definition 3.4:[25]}

Let $\left(R\left(I_{1}, I_{2}\right),+,.\right)$ be a refined neutrosophic ring and $P_{0}, P_{1}, P_{2}$ be ideals in the ring $\mathrm{R}$ then the set $P=$ $\left(P_{0}, P_{1} I_{1}, P_{2} I_{2}\right)=\left\{\left(a, b I_{1}, c I_{2}\right): a \in P_{0}, b \in P_{1}, c \in P_{2}\right\}$ is called a refined neutrosophic AH-ideal.

If $P_{0}=P_{1}=P_{2}$ then $P$ is called a refined neutrosophic AHS-ideal.

\section{Definition 3.5:[25]}

(a) Let $f: \mathrm{R}\left(I_{1}, I_{2}\right) \rightarrow \mathrm{T}\left(I_{1}, I_{2}\right)$ be an AHS-homomorphism. We define AH-Kernel of $\mathrm{f}$ by : AH - Kerf $=$ $\left\{\left(a, b I_{1}, c I_{2}\right) ; a, b, c \in \operatorname{Kerf}_{R}\right\}=\left(\operatorname{Kerf}_{R}, \operatorname{Kerf}_{R} I_{1}, \operatorname{Kerf}_{R} I_{2}\right)$

(b) Let $S=\left(S_{0}, S_{1} I_{1}, S_{2} I_{2}\right)$ be a subset of $R\left(I_{1}, I_{2}\right)$, we have: $f(S)=\left(f_{R}\left(S_{0}\right), f_{R}\left(S_{1}\right) I_{1}, f_{R}\left(S_{2}\right) I_{2}\right)=$ $\left\{\left(f_{R}\left(a_{0}\right), f_{R}\left(a_{1}\right) I_{1}, f_{R}\left(a_{2}\right) I_{2}\right) ; a_{i} \in S_{i}\right\}$

(c) Let $\mathrm{S}=\left(S_{0}, S_{1} I_{1}, S_{2} I_{2}\right)$ be a subset of $T\left(I_{1}, I_{2}\right)$, we have:

$f^{-1}(S)=\left(f_{T}^{-1}\left(S_{0}\right), f_{T}^{-1}\left(S_{1}\right) I_{1}, f_{T}^{-1}\left(S_{2}\right) I_{2}\right)$.

\section{Theorem 3.1:[19]}

Let $(R,+, \times)$ be a ring and $R(I), R\left(I_{1}, I_{2}\right)$ be the related neutrosophic ring and refined neutrosophic ring respectively,

(a) There is a ring homomorphism $f: R\left(I_{1}, I_{2}\right) \rightarrow R(I)$.

(b) The additive group $(\operatorname{Ker} f,+)$ is isomorphic to the additive group $(R,+)$.

\section{Theorem 3.2:[19]}

Let $R$ be a ring, where $\operatorname{Char}(R)=2$, there is a subring of $R\left(I_{1}, I_{2}\right)$ such as $K$ with property $K \cong R$; $\mathrm{R}\left(I_{1}, I_{2}\right) / K \cong R(I)$.

\section{Example 3.2:}

Let $R=Z_{2}$, R is a ring with respect to addition and multiplication modulo 2, we have $\operatorname{Char}(R)=2$. 
$\mathrm{R}\left(I_{1}, I_{2}\right)=\left\{\left(a, b I_{1}, c I_{2}\right) ; a, b, c \in Z_{2}\right\}$ is the related refined neutrosophic ring.

$R(I)=\left\{a+b I ; a, b \in Z_{2}\right\}$ is the related neutrosophic ring.

Let $f$ be the ring homomorphism defined above, we have $K=\operatorname{Ker} f=\left\{\left(0, b I_{1},-b I_{2}\right) ; b \in Z_{2}\right\}=$ $\left\{(0,0,0),\left(0, I_{1}, I_{2}\right)\right\} \cong R$.

$\mathrm{R}\left(I_{1}, I_{2}\right) / K=\left\{K,(1,0,0)+K,\left(0, I_{1}, 0\right)+K,\left(1,0, I_{2}\right)+K\right\} \cong R(I)$.

$\mathrm{O}\left(\mathrm{R}\left(I_{1}, I_{2}\right)\right)=8, O(R(I))=4, O(R)=2$, we find $O\left(\mathrm{R}\left(I_{1}, I_{2}\right)\right) / O(R)=O(R(I))=4$.

\section{Theorem 3.3:[19]}

Let $R$ be a ring, there is a subring of $R(I)$ say $K$ with property $K \cong R ; \mathrm{R}(I) / K \cong R$.

\section{Definition 3.6:[25]}

Let $\left(R\left(I_{1}, I_{2}\right),+,.\right)$ be a refined neutrosophic ring and $P=\left(P_{0}, P_{1} I_{1}, P_{2} I_{2}\right)$ be an AH-ideal then :

(a) We say that $P$ is a weak prime AH-ideal if $P_{i} ; i \in\{0,1,2\}$ are prime ideals in $R$.

(b) We say that $P$ is a weak maximal AH-ideal if $P_{i} ; i \in\{0,1,2\}$ are maximal ideals in $R$.

(c) We say that $P$ is a weak principal AH-ideal if $P_{i} ; i \in\{0,1,2\}$ are principal ideals in $R$.

(d) We define AH-factor as : $R\left(I_{1}, I_{2}\right) / P=\left(R / P_{0}, R / P_{1} I_{1}, R / P_{0} I_{2}\right)$.

\section{Example 3.3:}

Let $R=\left(Z_{6},+,.\right), T=\left(Z_{10},+,.\right)$ be two rings and $f$ is the AHS-homomorphism defined, we have the following :

(a) $P_{0}=\{0,2,4\}, P_{1}=\{0,3\}$ are two ideals in $Z_{6}$ thus $P=\left(P_{0}, P_{0} I_{1}, P_{1} I_{2}\right)$ is an AH-ideal of $R\left(I_{1}, I_{2}\right)$

(b) $f(P)=\left(f\left(P_{0}\right), f\left(P_{0}\right) I_{1}, f\left(P_{1}\right) I_{2}\right)=\left\{(0,0,0),\left(0,0,5 I_{2}\right)\right\}$ is a refined neutrosophic AH-ideal in $T\left(I_{1}, I_{2}\right)$.

(c) $Q_{0}=\{0,2,4,6,8\}$ is a maximal ideal in $Z_{10}$ and $f_{T}^{-1}\left(Q_{0}\right)=\{0,2,4\}$, so $Q=\left(Q_{0}, Q_{0} I_{1}, Q_{0} I_{2}\right)$ is a weak maximal refined neutrosophic AHS-ideal in $T\left(I_{1}, I_{2}\right)$, we have $f^{-1}(Q)=\left(\{0,2,4\},\{0,2,4\} I_{1},\{0,2,4\} I_{2}\right)$ is a weak maximal refined neutrosophic AHS-ideal in $R\left(I_{1}, I_{2}\right)$.

\section{Example 3.4:}

(a) In the ring $(Z,+,),. P=<3>, Q=<2>$ are two prime and maximal ideals, thus $M=\left(P, Q I_{1}, Q I_{2}\right)=$ $\left\{\left(3 a, 2 b I_{1}, 2 c I_{2}\right) ; a, b, c \in Z\right\}$ is a weak maximal/prime refined neutrosophic AH-ideal of $\left(Z\left(I_{1}, I_{2}\right)\right.$, + ,.).

(b)The map $f_{Z}: Z \rightarrow Z_{6} ; f(a)=a \bmod 6$ is a homomorphism so the related refined neutrosophic AHShomomorphism is 
$f: Z\left(I_{1}, I_{2}\right) \rightarrow Z_{6}\left(I_{1}, I_{2}\right) ; f\left(a, b I_{1}, c I_{2}\right)=\left(a \bmod 6,(b \bmod 6) I_{1},(c \bmod 6) I_{2}\right), \mathrm{AH}-\operatorname{ker} f=\left(6 \mathrm{Z}, 6 \mathrm{ZI} I_{1}\right.$, $\left.6 \mathrm{ZI}_{2}\right) \leq M$ since $6 \mathrm{Z} \leq P, Q$.

(c) $f(M)=\left(\{0,3\},\{0,2,4\} I_{1},\{0,2,4\} I_{2}\right)$ is a weak maximal/prime refined neutrosophic AH-ideal of $Z_{6}\left(I_{1}, I_{2}\right)$.

\section{Theorem 3.4:[25]}

Let $f: R\left(I_{1}, I_{2}\right) \rightarrow T\left(I_{1}, I_{2}\right)$ be an AHS-homomorphism, we have :

(a)AH-Ker(f) is an AHS-ideal of $R\left(I_{1}, I_{2}\right)$.

(b) If $P$ is a refined neutrosophic AH-ideal of $\mathrm{R}\left(I_{1}, I_{2}\right)$, then $f(P)$ is a refined neutrosophic AH-ideal of $\mathrm{T}\left(I_{1}, I_{2}\right)$.

(c) If $P$ is a refined neutrosophic AHS-ideal of R $\left(I_{1}, I_{2}\right)$, then $f(P)$ is a refined neutrosophic AHS-ideal of $T\left(I_{1}, I_{2}\right)$.

Open problem (2): Describe the algebraic structure of the group of units of any refined neutrosophic ring?

Open problem (3): Determine the form of primes in the refined neutrosophic ring of integers.

Open problem (4): Is Euler's Theorem still true in the refined neutrosophic ring of integers?.

Open problem (5): Is the fundamental Theorem of arithmetic still true in the refined neutrosophic ring of integers?.

\section{4. $n$-Refined neutrosophic rings}

Definition 4.1:[20]

Let $(R,+, \times)$ be a ring and $I_{k} ; 1 \leq k \leq n$ be $n$ indeterminacies. We define $R_{n}(I)=\left\{a_{0}+a_{1} I+\cdots+\right.$ $\left.a_{n} I_{n} ; a_{i} \in R\right\}$ to be $n$-refined neutrosophic ring. Addition and multiplication on $R_{n}(I)$ are defined as:

$\sum_{i=0}^{n} x_{i} I_{i}+\sum_{i=0}^{n} y_{i} I_{i}=\sum_{i=0}^{n}\left(x_{i}+y_{i}\right) I_{i}, \sum_{i=0}^{n} x_{i} I_{i} \times \sum_{i=0}^{n} y_{i} I_{i}=\sum_{i, j=0}^{n}\left(x_{i} \times y_{j}\right) I_{i} I_{j}$,

where $\times$ is the multiplication defined on the ring $R$.

It is easy to see that $R_{n}(\mathrm{I})$ is a ring in the classical concept and contains a proper ring $R$.

\section{Definition 4.2:[20]}

Let $R_{n}(\mathrm{I})$ be an $n$-refined neutrosophic ring, it is said to be commutative if $x y=y x$ for each $x, y \in R_{n}(\mathrm{I})$, if there is $1 \in R_{n}(\mathrm{I})$ such $1 . x=x .1=x$, then it is called an $n$-refined neutrosophic ring with unity.

\section{Definition 4.3:[20]}


(a) Let $R_{n}(I)$ be an $n$-refined neutrosophic ring and $P=\sum_{i=0}^{n} P_{i} I_{i}=\left\{a_{0}+a_{1} I+\cdots+a_{n} I_{n}: a_{i} \in P_{i}\right\}$ where $P_{i}$ is a subset of $\mathrm{R}$, we define $\mathrm{P}$ to be an AH-subring if $P_{i}$ is a subring of $R$ for all $i$, AHS-subring is defined by the condition $P_{i}=P_{j}$ for all $i, j$.

(b) $P$ is an AH-ideal if $P_{i}$ is an two sides ideal of $R$ for all $i$, the AHS-ideal is defined by the condition $P_{i}=P_{j}$ for all $i, j$.

(c) The AH-ideal $P$ is said to be null if $P_{i}=R$ or $P_{i}=\{0\}$ for all $i$.

\section{Theorem 4.1:[20]}

Let $R_{n}(I)$ be an $n$-refined neutrosophic ring and $P$ is an AH-ideal, $(P,+)$ is an abelian neutrosophic group with $k \leq n$ and r.p $P$ for all $p \in P$ and $r \in R$.

\section{Theorem 4.2:[20]}

Let $R_{n}(\mathrm{I})$ be an $n$-refined neutrosophic ring and $P=\sum_{i=0}^{n} P_{i} I_{i}, Q=\sum_{i=0}^{n} Q_{i} I_{i}$ be two AH- ideals then $P+Q, P \cap Q$ are AH-ideals. If $P, Q$ are AHS-ideals then $P+Q, P \cap Q$ are AHS-ideals.

\section{Theorem 4.3:[20]}

Let $R_{n}(\mathrm{I})$ be an $n$-refined neutrosophic ring and $P=\sum_{i=0}^{n} P_{i} I_{i}$ be an AH-ideal, we define AH-factor $R(I) / P$ $=\sum_{i=0}^{n}\left(R / P_{i}\right) I_{i}=\sum_{i=0}^{n}\left(x_{i}+P_{i}\right) I_{i} ; x_{i} \in R$.

\section{Definition 4.4:[20]}

a) Let $R_{n}(\mathrm{I}), T_{n}(\mathrm{I})$ be two $n$-refined neutrosophic rings respectively, and $f_{R}: R \rightarrow T$ be a ring homomorphism. We define $n$-refined neutrosophic AHS-homomorphism as :

$$
f: R_{n}(\mathrm{I}) \rightarrow T_{n}(\mathrm{I}) ; f\left(\sum_{i=0}^{n} x_{i} I_{i}\right)=\sum_{i=0}^{n} f_{R}\left(x_{i}\right) I_{i} .
$$

(b) $f$ is an n-refined neutrosophic AHS-isomorphism if it is a bijective n-refined neutrosophic AHShomomorphism.

(c) $\mathrm{AH}-\operatorname{Ker} f=\sum_{i=0}^{n} \operatorname{Ker}\left(f_{R}\right) I_{i}=\left\{\sum_{i=0}^{n} x_{i} I_{i} ; x_{i} \in \operatorname{Ker} f_{R}\right\}$.

\section{Theorem 4.4:[20]}

Let $R_{n}(\mathrm{I}), T_{n}(\mathrm{I})$ be two n-refined neutrosophic rings respectively and $f$ be an n-refined neutrosophic AHS-homomorphism $f: R_{n}(\mathrm{I}) \rightarrow T_{n}(\mathrm{I})$. Then

(a) If $P=\sum_{i=0}^{n} P_{i} I_{i}$ is an AH- subring of $R_{n}(\mathrm{I})$ then $f(P)$ is an AH- subring of $T_{n}(\mathrm{I})$,

(b) If $P=\sum_{i=0}^{n} P_{i} I_{i}$ is an AHS- subring of $R_{n}(\mathrm{I})$ then $f(P)$ is an AHS- subring of $T_{n}(\mathrm{I})$,

(c) If $P=\sum_{i=0}^{n} P_{i} I_{i}$ is an AH-ideal of $R_{n}(\mathrm{I})$ then $f(P)$ is an AH-ideal of $\mathrm{f}\left(R_{n}(\mathrm{I})\right)$,

(d) $P=\sum_{i=0}^{n} P_{i} I_{i}$ is an AHS-ideal of $R_{n}(\mathrm{I})$ then $f(P)$ is an AHS-ideal of $\mathrm{f}\left(R_{n}(\mathrm{I})\right)$, 
(e) $R_{n}(\mathrm{I}) / A H-K e r(f)$ is AHS - isomorphic to $f(R(I))$,

(f) Inverse image of an AH-ideal $P$ in $T_{n}(\mathrm{I})$ is an AH-ideal in R(I).

\section{Example 4.1:}

Let $R=Z_{8}$ be a ring with addition and multiplication modulo 8 .

(a) 3-refined neutrosophic ring related with $R$ is $Z_{83}(\mathrm{I})=\left\{\mathrm{a}+b I_{1}+c I_{2}+d I_{3} ; a, b, c, d \in Z_{8}\right\}$.

(b) $\mathrm{P}=\{0,4\}$ is an ideal of $\mathrm{R}, \sqrt{P}=\{0,2,4,6\}, M=P+P I_{1}+P I_{2}+P I_{3}$ is an AHS-ideal of $Z_{83}(\mathrm{I})$,

$A H-\operatorname{Rad}(M)=\sqrt{P}+\sqrt{P} I_{1}+\sqrt{P} I_{2}+\sqrt{P} I_{3}$ which is an AHS-ideal of $Z_{8_{3}}(\mathrm{I})$.

\section{Example 4.2:}

Let $\mathrm{R}=Z_{2}$ be the ring of integers modulo 2, let $n=3$. The corresponding 3-refined neutrosophic ring is $Z_{2_{3}}(\mathrm{I})=\left\{0,1, I_{1}, I_{2}, I_{3}, 1+I_{1}, 1+I_{2}, 1+I_{3}, I_{1}+I_{2}, I_{1}+I_{3}, I_{1}+I_{2}+I_{3}, I_{2}+I_{3}, 1+I_{1}+I_{2}+I_{3}, 1+I_{2}+\right.$ $\left.I_{3}, 1+I_{1}+I_{3}, 1+I_{1}+I_{2}\right\}$.

Open problem (6): Describe the algebraic structure of the group of units in any $n$-refined neutrosophic ring.

Open problem (7): Determine the necessary and sufficient condition for the idempotency in any $n$ refined neutrosophic ring.

Open problem (8): Define AH-homomorphisms, do they have an $n$-refined neutrosophic ring structure?

Open problem (9): Define number theoretical concepts over the $n$-refined neutrosophic ring of integers.

Open problem (10): Determine an algorithm to solve Diophantine equations (especially Pell's equation) in the $n$-refined neutrosophic ring of integers.

Open problem (11): Determine the form of primes, Euler's Theorem in the $n$-refined neutrosophic ring of integers.

\section{Neutrosophic complex numbers rings and fields}

\section{Definition 5.1:[28]}

Let $R$ be the field of real numbers, neutrosophic complex numbers set is defined as $C(R \cup I)=\{a+b I+c i+d I i ; a, b, c, d \in R\}$

\section{Definition 5.2:[28]}

Let $Q$ be the field of rational numbers, rational neutrosophic complex numbers set is defined as $C(Q \cup I)=\{a+b I+c i+d I i ; a, b, c, d \in Q\}$. 


\section{Definition 5.3:[28]}

Let $Z$ be the ring of integer numbers, integer neutrosophic complex numbers set is defined as

$C(Z \cup I)=\{a+b I+c i+d I i ; a, b, c, d \in Z\}$.

\section{Definition 5.4:[28]}

Let $Z_{n}$ be the ring of integers modulo $n$, the finite ring of complex numbers is defined as

$C\left(Z_{n}\right)=\left\{a+b i ; i^{2} \equiv-1(\bmod n)\right\}$.

\section{Definition 5.5:[28]}

We define the neutrosophic ring of finite complex numbers as follows:

$C\left(Z_{n} \cup I\right)=\left\{a+b i+c I+d i I ; i^{2} \equiv-1(\bmod n)\right\}$.

\section{Remark 5.1:[28]}

Neutrosophic complex numbers is considered as a generalization of classical complex numbers.

\section{Example 5.1:}

Let $Z_{3}$ be the ring of integers modulo 3, then

$C\left(Z_{3}\right)=\left\{a+b i ; i^{2} \equiv-1(\bmod 3)\right\}=\{0,1,2, i, 1+i, 2+i, 2 i, 1+2 i, 2+2 i\}$.

\section{Example 5.2:}

$C\left(Z_{3} \cup I\right)=\{a+b i+c I+d i I ; a, b, c \in\{0,1,2\}\}$.

Open problem (12): Can $C(<Z \cup I>)$ be a Smarandache ring?

Open problem (13): Can $C(<R \cup I>)$ have irreducible polynomials?.

Open problem (14): Determine the irreducible polynomials over $C(\langle Q \cup I\rangle)$ ?.

Open problem (15): Find irreducible polynomials in $C(<Z \cup I\rangle)[x]$ ?. Is every ideal in $C(<Z \cup I\rangle)$ is principal?.

Open problem (16): Can one say for all polynomials with complex neutrosophic coefficients $C(\langle R \cup I\rangle)$ is algebraically closed?.

Open problem (17): Describe the group of units structure in $C(<Z \cup I>), C(<R \cup I\rangle), C(<Q \cup$ $I>$ ).

Open problem (18): Find zero divisors and units in $C\left(Z_{24}\right)$. In general, determine the sufficient and necessary condition to any element to be a zero divisor. 
Open problem (19): Find the form of ideals and maximal ideals in $\mathrm{C}\left(\left\langle Z_{n} \cup \mathrm{I}\right\rangle\right)$.

Open problem (20): Find a necessary and sufficient condition for a complex modulo integers ring $S=C\left(Z_{n}\right)$ to have ideal I such that $C\left(Z_{n}\right) / I$ is never a field.

Open problem (21): Determine the algebraic structure of the group of units of the ring $C\left(\left\langle Z_{n} \cup \mathrm{I}\right\rangle\right)$.

Open problem (22): Find the relationship between algebraic elements in any neutrosophic ring and its corresponding classical ring.

Open Problem (23): Find the relationship between algebraic elements in any refined neutrosophic ring and its corresponding classical ring.

Open Problem (24): Find the relationship between algebraic elements in any $n$-refined neutrosophic ring and its corresponding classical ring.

Open Problem (25): Describe the algebraic structure of the group of units in the ring $C\left(Z_{n}\right)$.

\section{Conclusion}

In this article, we have recalled some important and new trends in algebra, where the concept of neutrosophic ring, refined neutrosophic ring, $n$-refined neutrosophic ring, and neutrosophic complex numbers' ring are discussed.

Also, we have presented a review about the most interesting 25 open problems about these concepts. Many of these questions were asked by Smarandache and Kandasamy in [28] about the structure of neutrosophic complex ring numbers. These open questions will give a wide push to theoretical studies if they have been solved.

We presented many interesting examples to clarify the validity of these concepts and to clarify their structures.

Funding:: This research received no external funding

Conflicts of Interest: The authors declare no conflict of interest

\section{References}

[1] Smarandache, F., " A Unifying Field in Logics: Neutrosophic Logic, Neutrosophy, Neutrosophic Set, Neutrosophic Probability", American Research Press. Rehoboth, 2003.

[2].Abdel-Basset, M., Gamal, A., Son, L. H., and Smarandache, F.,"A Bipolar Neutrosophic Multi Criteria Decision Making Framework for Professional Selection", Applied Sciences, 10(4), 2020.

[3].Abdel-Basset, M., Mohamed, R., Zaied, A. E. N. H., Gamal, A., \& Smarandache, F. " Solving the supply chain problem using the best-worst method based on a novel Plithogenic model. In Optimization Theory Based on Neutrosophic and Plithogenic Sets", (pp. 1-19), Academic Press, 2020. 
[4].Abdel-Basset, Mohamed., "An integrated plithogenic MCDM approach for financial performance evaluation of manufacturing industries", Risk Management, pp. 1-27, 2020.

[5].Abdel-Basst, M., Mohamed, R., \& Elhoseny, M., " A novel framework to evaluate innovation value proposition for smart product-service systems. Environmental Technology \& Innovation", vol. 11, pp. 102-109, 2020.

[6].Abdel-Basst, Mohamed, Rehab Mohamed, and Mohamed Elhoseny.," < ? covid19?> A model for the effective COVID-19 identification in uncertainty environment using primary symptoms and CT scans", Health Informatics Journal, 2020 .

[7] Alhamido, R., and Gharibah, T., "Neutrosophic Crisp Tri-Topological Spaces", Journal of New Theory, Vol. 23 , pp.13-21. 2018.

[8] Edalatpanah. S.A., "Systems of Neutrosophic Linear Equations", Neutrosophic Sets and Systems, Vol. 33, pp. 92-104. 2020.

[9] Sankari, H., and Abobala, M., "Neutrosophic Linear Diophantine Equations With two Variables", Neutrosophic Sets and Systems, Vol. 38, pp. 22-30, 2020.

[10] Sankari, H., and Abobala, M." n-Refined Neutrosophic Modules", Neutrosophic Sets and Systems, Vol. 36, pp. 1-11. 2020.

[11] Alhamido, R., and Abobala, M., "AH-Substructures in Neutrosophic Modules", International Journal of Neutrosophic Science, Vol. 7, pp. 79-86 . 2020.

[12] Abobala, M., "AH-Subspaces in Neutrosophic Vector Spaces", International Journal of Neutrosophic Science, Vol. 6, pp. 80-86. 2020.

[13] Abobala, M.,. "A Study of AH-Substructures in $n$-Refined Neutrosophic Vector Spaces", International Journal of Neutrosophic Science", Vol. 9, pp.74-85. 2020.

[14] Hatip, A., Alhamido, R., and Abobala, M., "A Contribution to Neutrosophic Groups", International Journal of Neutrosophic Science", Vol. 0, pp. 67-76 . 2019.

[15] Abobala, M., " n-Refined Neutrosophic Groups I", International Journal of Neutrosophic Science, Vol. 0, pp. 27-34. 2020.

[16] Kandasamy, V.W.B., and Smarandache, F., "Some Neutrosophic Algebraic Structures and Neutrosophic N-Algebraic Structures", Hexis, Phonex, Arizona, 2006.

[17] Agboola, A.A.A., Akinola, A.D., and Oyebola, O.Y., " Neutrosophic Rings I" , International J.Mathcombin, Vol 4,pp 1-14. 2011

[18] Agboola, A.A.A., "On Refined Neutrosophic Algebraic Structures," Neutrosophic Sets and Systems, Vol.10, pp. 99-101. 2015.

[19] Abobala, M., "Classical Homomorphisms Between Refined Neutrosophic Rings and Neutrosophic Rings", International Journal of Neutrosophic Science, Vol. 5, pp. 72-75. 2020. 
[20] Smarandache, F., and Abobala, M., n-Refined neutrosophic Rings, International Journal of Neutrosophic Science, Vol. 5 , pp. 83-90, 2020.

[21] Kandasamy, I., Kandasamy, V., and Smarandache, F., "Algebraic structure of Neutrosophic Duplets in Neutrosophic Rings", Neutrosophic Sets and Systems, Vol. 18, pp. 85-95. 2018.

[22] Yingcang, Ma., Xiaohong Zhang ., Smarandache, F., and Juanjuan, Z., "The Structure of Idempotents in Neutrosophic Rings and Neutrosophic Quadruple Rings", Symmetry Journal (MDPI), Vol. 11. 2019.

[23] Kandasamy, V. W. B,. Ilanthenral, K., and Smarandache, F., "Semi-Idempotents in Neutrosophic Rings", Mathematics Journal (MDPI), Vol. 7. 2019.

[24] Abobala, M., On Some Special Substructures of Neutrosophic Rings and Their Properties, International Journal of Neutrosophic Science", Vol. 4, pp. 72-81, 2020.

[25] Abobala, M., "On Some Special Substructures of Refined Neutrosophic Rings", International Journal of Neutrosophic Science, Vol. 5, pp. 59-66. 2020.

[26] Smarandache, F., and Ali, M., "Neutrosophic Triplet Group", Neural. Compute. Appl. 2019.

[27] Sankari, H., and Abobala, M.," AH-Homomorphisms In neutrosophic Rings and Refined Neutrosophic Rings", Neutrosophic Sets and Systems, Vol. 38, pp. 101-112, 2020.

[28] Smarandache, F., and Kandasamy, V.W.B., " Finite Neutrosophic Complex Numbers",-Source: arXiv. 2011.

[29]. Suresh, R., and S. Palaniammal,. "Neutrosophic Weakly Generalized open and Closed Sets", Neutrosophic Sets and Systems, Vol. 33, pp. 67-77,. 2020.

[30] Sahin, M., Olgun, N., Uluçay, V., Kargın, A., and Smarandache, F. , "A New Similarity Measure Based on Falsity Value between Single Valued Neutrosophic Sets Based on the Centroid Points of Transformed Single Valued Neutrosophic Numbers with Applications to Pattern Recognition", Neutrosophic Sets and Systems, vol. 15, pp. 31-48. 2017.

[31] Ibrahim, M.A., Agboola, A.A.A, Badmus, B.S. and Akinleye, S.A., "On refined Neutrosophic Vector Spaces I", International Journal of Neutrosophic Science, Vol. 7, pp. 97-109. 2020.

[32] Ibrahim, M.A., Agboola, A.A.A, Badmus, B.S., and Akinleye, S.A., "On refined Neutrosophic Vector Spaces II", International Journal of Neutrosophic Science, Vol. 9, pp. 22-36. 2020.

[33] Abobala, M, " $n$-Cyclic Refined Neutrosophic Algebraic Systems Of Sub-Indeterminacies, An Application To Rings and Modules", International Journal of Neutrosophic Science, Vol. 12, pp. 81-95 . 2020.

[34] Smarandache, F., "Neutrosophic Set a Generalization of the Intuitionistic Fuzzy Sets", Inter. J. Pure Appl. Math., pp. 287-297. 2005. 
[35] M. Ali, F. Smarandache, M. Shabir and L. Vladareanu., "Generalization of Neutrosophic Rings and Neutrosophic Fields", Neutrosophic Sets and Systems, vol. 5, pp. 9-14, 2014.

[36] Anuradha V. S., "Neutrosophic Fuzzy Hierarchical Clustering for Dengue Analysis in Sri Lanka", Neutrosophic Sets and Systems, vol. 31, pp. 179-199. 2020.

[37] Olgun, N., and Hatip, A., "The Effect Of The Neutrosophic Logic On The Decision Making, in Quadruple Neutrosophic Theory And Applications", Belgium, EU, Pons Editions Brussels,pp. 238-253. 2020 .

[38] Zadeh, L. "Fuzzy Sets", Inform and Control, 8, pp.338-353. 1965.

[39] Turksen, I., "Interval valued fuzzy sets based on normal forms", Fuzzy Sets and Systems, 20, pp.191-210, 1986. 1986.

[40] Chalapathi, T., and Madhavi, L., "Neutrosophic Boolean Rings", Neutrosophic Sets and Systems, Vol. 33, pp. 57-66. 2020.

[41] Abobala, M., "Classical Homomorphisms Between n-refined Neutrosophic Rings", International Journal of Neutrosophic Science", Vol. 7, pp. 74-78. 2020.

[42] Agboola, A.A.A,. Akwu, A.D,. and Oyebo, Y.T., " Neutrosophic Groups and Subgroups", International .J .Math. Combin, Vol. 3, pp. 1-9. 2012.

[43] Smarandache, F., " $n$-Valued Refined Neutrosophic Logic and Its Applications in Physics", Progress in Physics, 143-146, Vol. 4, 2013.

[44] Adeleke, E.O., Agboola, A.A.A.,and Smarandache, F., "Refined Neutrosophic Rings I", International Journal of Neutrosophic Science, Vol. 2(2), pp. 77-81. 2020.

[45] Hatip, A., and Abobala, M., "AH-Substructures In Strong Refined Neutrosophic Modules", International Journal of Neutrosophic Science, Vol. 9, pp. 110-116 . 2020.

[46] Hatip, A., and Olgun, N., "On Refined Neutrosophic R-Module", International Journal of Neutrosophic Science, Vol. 7, pp.87-96. 2020.

[47] Chakraborty, A., Banik, B., Mondal, S.P., and Alam, S., "Arithmetic and Geometric Operators of Pentagonal Neutrosophic Number and its Application in Mobile Communication Service Based MCGDM Problem", Neutrosophic Sets and Systems, vol. 32, pp. 61-79. 2020.

[48] Smarandache F., and Abobala, M., " $n$-Refined Neutrosophic Vector Spaces", International Journal of Neutrosophic Science, Vol. 7, pp. 47-54. 2020.

[49] Sankari, H., and Abobala, M., "Solving Three Conjectures About Neutrosophic Quadruple Vector Spaces", Neutrosophic Sets and Systems, Vol. 38, pp. 70-77. 2020.

[50] Adeleke, E.O., Agboola, A.A.A., and Smarandache, F., "Refined Neutrosophic Rings II", International Journal of Neutrosophic Science, Vol. 2(2), pp. 89-94. 2020. 
[51] Abobala, M., On Refined Neutrosophic Matrices and Their Applications In Refined Neutrosophic Algebraic Equations, Journal Of Mathematics, Hindawi, 2021

[52] Abobala, M., A Study of Maximal and Minimal Ideals of n-Refined Neutrosophic Rings, Journal of Fuzzy Extension and Applications, Vol. 2, pp. 16-22, 2021.

[53] Abobala, M., " Semi Homomorphisms and Algebraic Relations Between Strong Refined Neutrosophic Modules and Strong Neutrosophic Modules", Neutrosophic Sets and Systems, Vol. 39, 2021.

[54] Abobala, M., "On Some Neutrosophic Algebraic Equations", Journal of New Theory, Vol. 33, 2020.

[55] Abobala, M., On The Representation of Neutrosophic Matrices by Neutrosophic Linear Transformations, Journal of Mathematics, Hindawi, 2021.

[56] Abobala, M., "On Some Algebraic Properties of n-Refined Neutrosophic Elements and n-Refined Neutrosophic Linear Equations", Mathematical Problems in Engineering, Hindawi, 2021

[57] Kandasamy V, Smarandache F., and Kandasamy I., Special Fuzzy Matrices for Social Scientists . Printed in the United States of America,2007, book, 99 pages.

[58] Khaled, H., and Younus, A., and Mohammad, A., " The Rectangle Neutrosophic Fuzzy Matrices", Faculty of Education Journal Vol. 15, 2019. (Arabic version).

[59] Abobala, M., Partial Foundation of Neutrosophic Number Theory, Neutrosophic Sets and Systems, Vol. 39, 2021.

[60] F. Smarandache, Neutrosophic Theory and Applications, Le Quy Don Technical University, Faculty of Information technology, Hanoi, Vietnam, $17^{\text {th }}$ May 2016.

[61] Sankari, H, and Abobala, M., " On A New Criterion For The Solvability of non Simple Finite Groups and m-Abelian Solvability, Journal of Mathematics, Hindawi, 2021.

[62] Abobala, M., "On Some Special Elements In Neutrosophic Rings and Refined Neutrosophic Rings", Journal of New Theory, vol. 33, 2020.

[63] Sankari, H, and Abobala, M, " A Contribution to m-Power Closed Groups", UMM-Alqura University Journal for Applied Sciences, KSA, 2020. 